\title{
A400M. Flares trajectories calculation from a chase aircraft
}

\author{
Francisca Coll Herrero ${ }^{1}$, Israel Lopez Herreros ${ }^{2}$ \\ 1: Airbus, francisca.coll@airbus.com \\ 2: Airbus, Israel.Lopez@airbus.com \\ Flight Test - Airbus Defence\&Space, Avd. John Lennon s/n, 28906 Getafe (Spain)
}

\begin{abstract}
The A400M defensive aids Sub-System (DASS) includes a radar warning receiver, missile launch and approach warning, and chaff and flare decoy dispensers.
\end{abstract}

The analysis of the safe separation of flares from A400M within its flight envelope is required as part of the certification process of A400M DASS.

This process includes the validation of the model used by the Aerodynamics Department to calculate the theoretical flare trajectories.

During 2017, it was required to validate the trajectory ejected from dispersers located on the nose of the aircraft, and in this case it was necessary to use external cameras installed in a chase aircraft to cover the completed trajectory.

The aim of this document is to present a methodology used to calculate a real case of flares trajectories on A400M ejected from front dispensers using 2 external cameras installed in a chase aircraft.

Keywords: DASS, safe separation High Speed Cameras, Photogrammetry, HSV, A400M, camera calibration, FollowMe, external cameras.

\section{Introduction}

The analysis of the safe separation of flares from A400M is required as part of the certification process of A400M DASS (Ref.1).

In order to validate the theoretical model used by the Aerodynamics Department several safe separation flight tests had to be performed.

Initially only dispensers placed in the rear wing fairing (WR $11 \& 12$ ) and especially in the rear part of the sponsors (SP $13 \& 14$ ) were selected to perform the tests as the most critical ones (Higher risk of impact, HTP) .

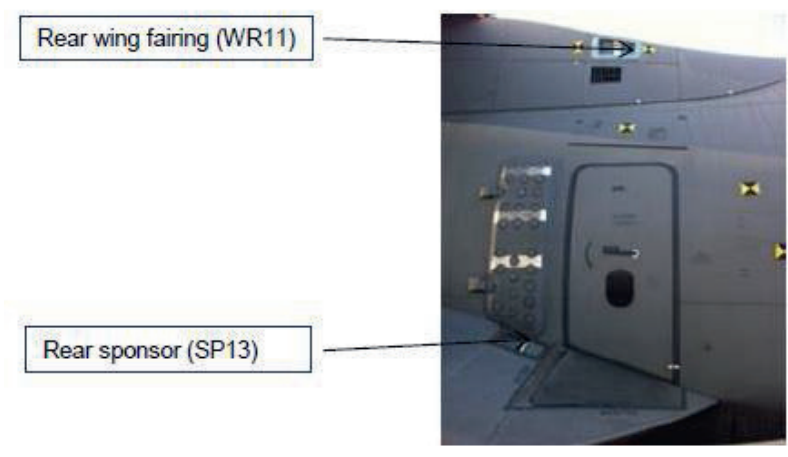

Figure 1.Dispensers

The trajectories are calculated by applying Photogrammetry techniques to the films extracted from onboard High Speed (HSV) cameras (Vannier -AOS, $1024 \times 768$ resolution, 200 frames per second).

Four synchronized cameras are needed to cover all theoretical paths, two cameras for the initial part and two for the final part of the path.

- $\mathrm{C} 3 \mathrm{~S}$, installed under the left wing, close to the tip

- HS3, installed on a balcony in the place of the last left side window.

- J1S, installed under the left HTP

- HS4, installed on the left wing- centre fuselage
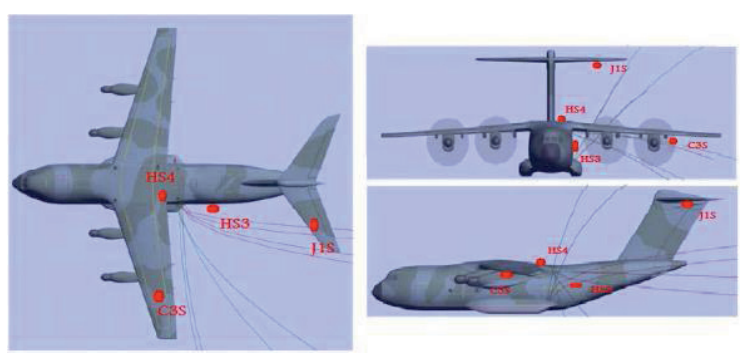

Figure 2. theoretical trajectories

The trajectory is calculated by sections, J1S and C3S for the initial part and HS4 and HS3 for the rest of the path. 


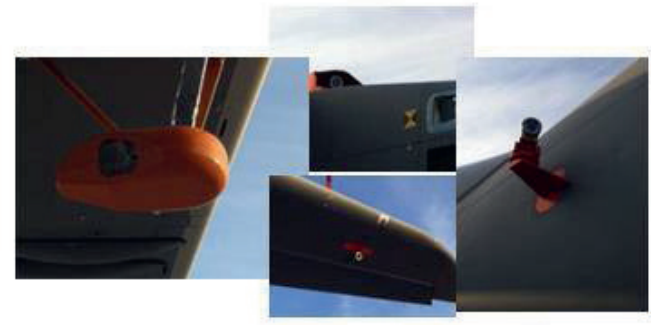

Figure 3 :HSV cameras

The Flight Physics Department gives theoretical paths of flares in different flight conditions.

Using the 3D-studio program, the cameras are positioned in order to cover all paths.

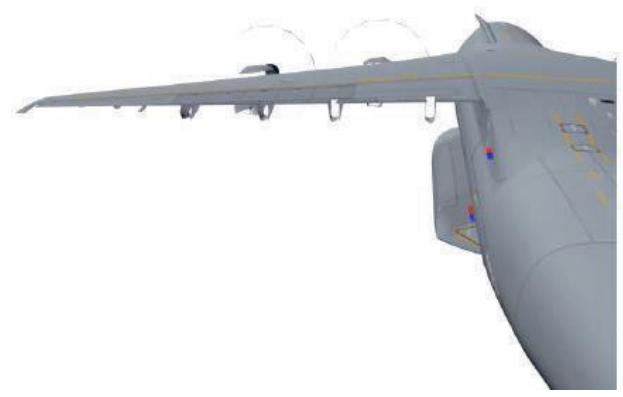

Figure 4.Image from J1S using 3D studio

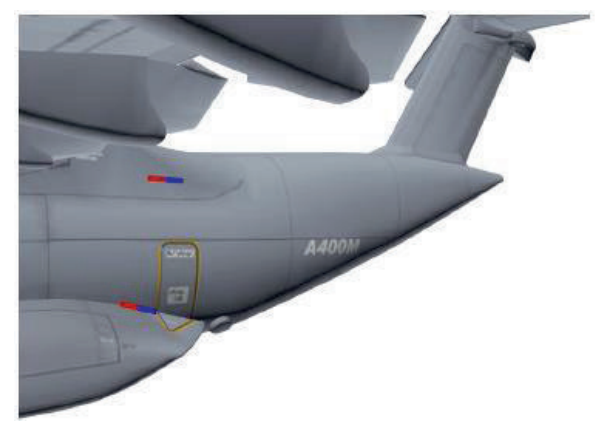

Figure 5.Image from C3S using 3D studio

During 2017, the validation of the flares trajectories from all the dispensers has been required.

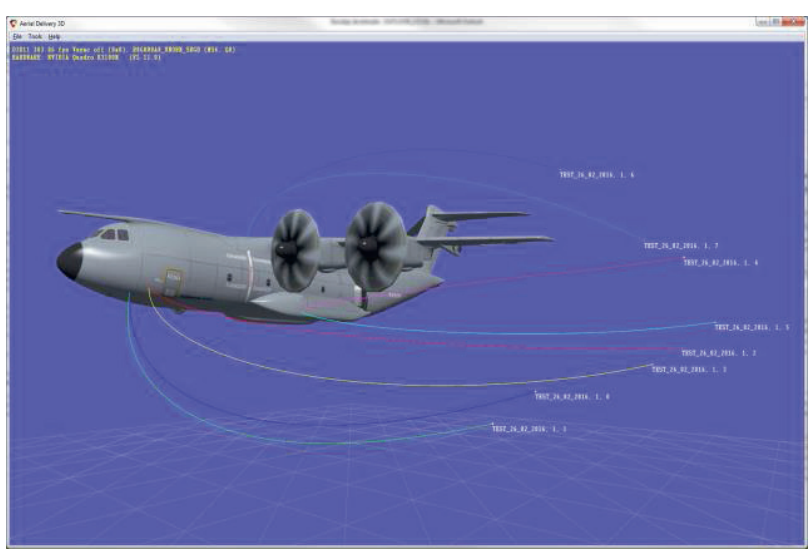

Figure 6.flares trajectories from all the dispensers
After a detailed study from our team, the following findings were made:

- It is impossible to calculate the trajectories of the flares using the cameras currently installed on the A $400 \mathrm{M}$, even changing the orientation of the cameras.

- It is practically impossible to calculate the trajectories of the flares from the dispensers located on the nose of the aircraft with cameras installed anywhere in the aircraft.

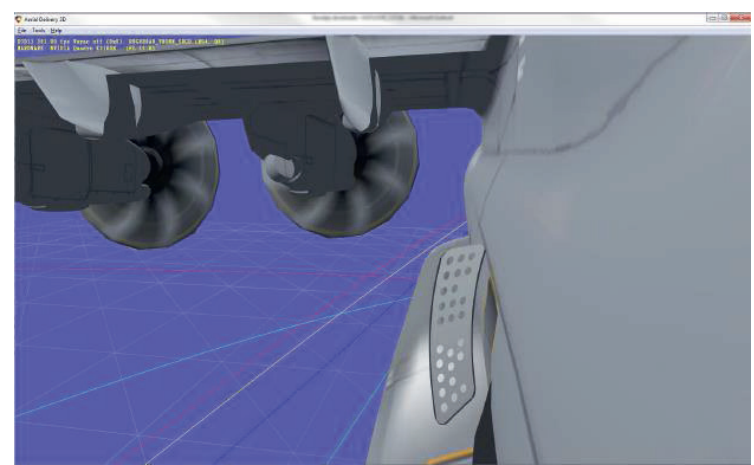

Figure 7.Image from HS3 changing the camera orientation

The aim of this document is to present a new methodology used to calculate a real case of flares trajectories on A400M ejected from front dispensers using 2 cameras installed in a chase aircraft.

During the Flight Tests campaign of safe separation, all the flares will be ejected from the left dispensers due to the symmetry of the aircraft.

The trajectories are calculated using programs developed by FT analysis Tools team of Airbus defense and space.

\section{Propose solution by FT Analysis Tools}

As mentioned in the previous chapter, it is practically impossible to calculate the trajectories of the flares ejected from the dispensers located on the nose of the aircraft with the cameras installed anywhere in the aircraft.

The solution proposed by FT Analysis Tools department was:

- $\quad$ To use a chase aircraft with 2 synchronized Full HD cameras installed using a tripod, in the right part of the cabin. 
- The airplane would fly in parallel to the A400M (security distance around 70 meters) in front of the dispenser to be tested.

- $\quad$ The proposed chase aircraft are the A310 (BOOM), C295 or A400M.

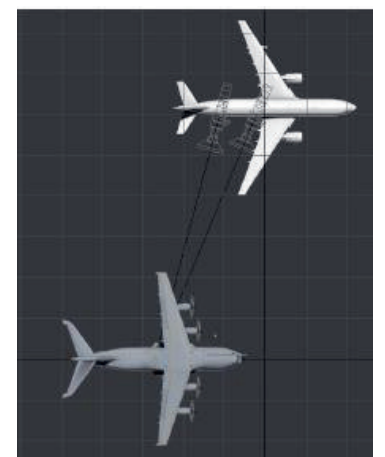

Figure 8. Schematic representation of FT Analysis Tools solution

\section{Background .Photogrammetry proces}

To calculate the trajectory of an object, photogrammetry procces is divided in to the followings steps: to define Aircraft System Reference, camera calibration, camera positioning and 3D trajectory calculation.

\subsection{Aircraft System Reference}

In the case of this report, the flares trajectory coordinates are given with respect to A400M coordinate system.

To define this AC coordinate system it is necessary to know the coordinates of at least 3 A400M reference markers, FTI design office provides us with this information.

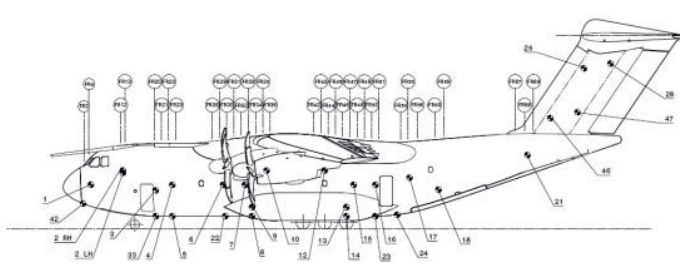

Figure 9.Reference marks on A400M

The figure 10 shows the references marks used in the analysis.

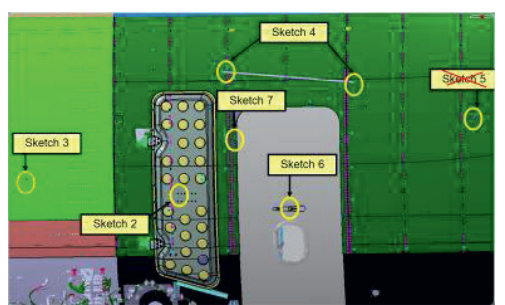

Figure 10.Reference marks used

\subsubsection{Tachymeter}

The tachymeter will be used to measure the exact coordinates of the reference marks ( Ref. 2).

With aid from a telemeter, the positions of the reference points are measured in telemeter coordinates and afterwards, transformed according to aircraft system reference.

Some stickers are used to mark points of interest to be measured with the tachymeter and transform to AC axes system through the reference points.

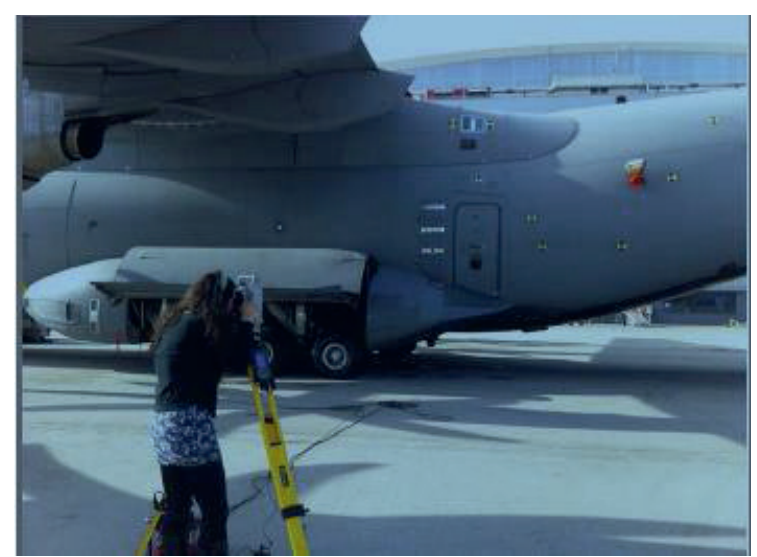

Figure 11. Aircrfat sistem reference

\subsection{Camera calibration}

Camera calibration, the aim of the calibration process is to obtain the intrinsic optical parameters of the cameras, more specifically of its lens, like focal length, principal point and distortion of the lens.

Matlab® Calibration Toolbox is used to determine the calibration (Ref. 3).

To calibrate a camera, the first step is to take pictures of a checked board (Figure 12). Several shots moving the board to different distances and angles are taken.
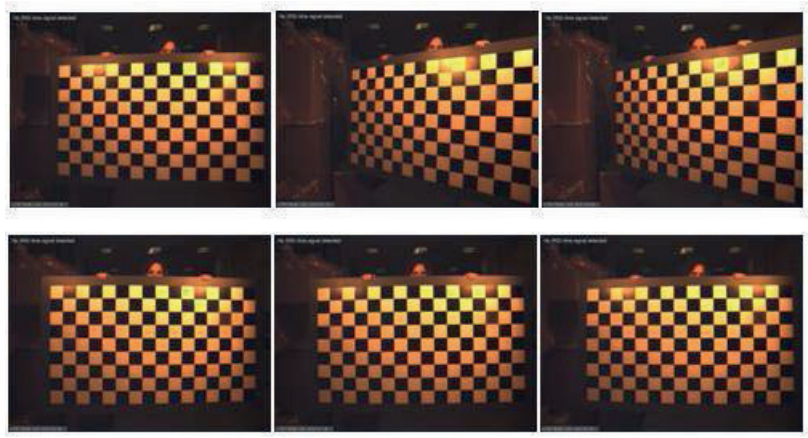

Figure 12.camera calibration 


\subsection{Cameras positioning}

Cameras positioning, the aim of this process is to calculate the position (X, Y, Z coordinates) and spatial alignment (roll, pitch, yaw) to respect Aircraft System Reference.

For this process, special stickers are used as reference marks by placing them inside the cameras' visible field.

\subsection{D trajectory calculation}

With the information obtained in the previous steps and the $2 \mathrm{D}$ coordenates of the object in the image, the $3 \mathrm{D}$ object coordenates are calculated using different algorithms for one or two cameras solution.

In the case of flares trajectories, the flares is considered as a point, therefore the algorithm used is two camera solution.

Both the camera position and the flare trajectory are calculated using in-house Software named FollowMe .This software, developed in Matlab®, is based on photogrammetry algorithms and automatic image recognition techniques integrated into a graphical interface to ease the selection of the reference marks.

\section{Mathematical solution}

Two possible mathematical solutions were considered to calculate the flare's trajectories ejected from nose dispenser.

For each frame of the film, were placed on A400M big stickers to be clearly seen from the chase cameras:

a) To calculate the two camera positions to respect A400M System Reference. The flare trajectory is calculated using the two camera solution for mobile cameras.

b) To calculate the transformation matrix between both aircrafts (chase and A400M). The flare trajectory is initially calculated to respect chase System reference and finally transformed to A400M System reference using the corresponding transformation matrix. This was the algorithm used in the analysis.

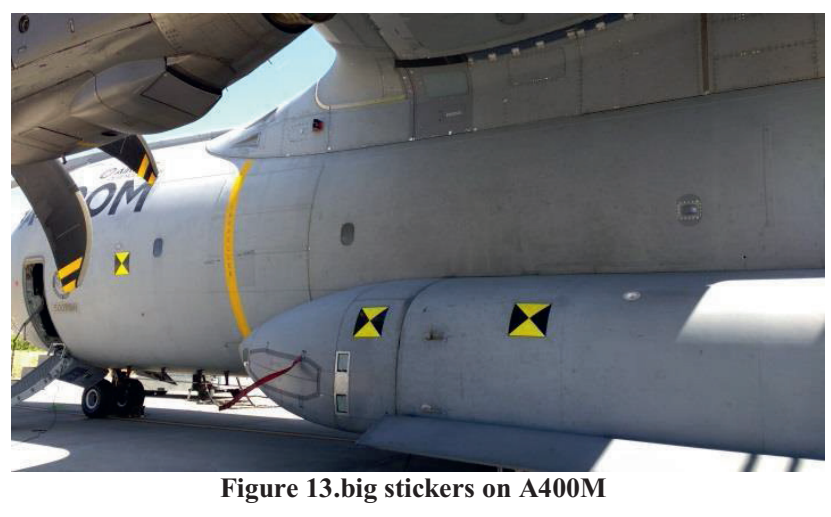

$5 \quad$ Validation and accuracy

The validation was made using FollowMe software to calculate flare 3D trajectory and 3DS Studio Max to study the scenario and the generation of the video/images.

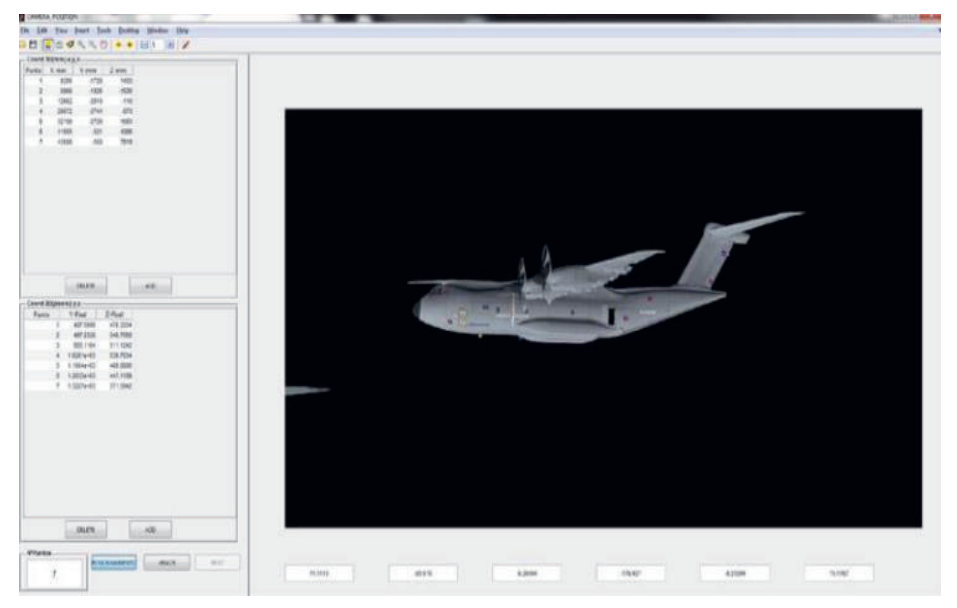

Figure 14 .FollowMe software

From the 3D theoretical flares trajectories and the cameras positions, 2D coordinates of the stickers and flares are calculated.

Using these theoretical 2D positions, and applying the algorithm, the obtained 3D trajectory is perfect.

In order to study the accuracy of the algorithm, it is considered 0.3 pixel error in the selection of the sctickers and the flare.

In both of the mathematical solutions, the error is less than 0.1 meter, therefore both techniques can be used for the flare trajectory calculation. 
6 Pros and Cons

\begin{tabular}{|l|l|}
\hline Pros & Cons \\
\hline $\begin{array}{l}\text { Not required additional } \\
\text { camera installation in A400M. }\end{array}$ & $\begin{array}{l}\text { Calculate cameras position in } \\
\text { each frame or transform } \\
\text { matrix. }\end{array}$ \\
\hline $\begin{array}{l}\text { Recording all left flare } \\
\text { trajectories in one flight and } \\
\text { one position }\end{array}$ & Chase aircraft required \\
\hline $\begin{array}{l}\text { COT cameras and easy } \\
\text { installation using tripod }\end{array}$ & \\
\hline $\begin{array}{l}\text { Two cameras for all the flare } \\
\text { trajectory }\end{array}$ & \\
\hline
\end{tabular}

\section{$7 \quad$ Flight Test campaign}

As part of the activities required for validating the A400M flare models of the flare types specified within DASS Certification Plan, a flight test campaign is scheduled in May 2017.

Those tests that consist in ejecting a flare from the rear sponson and wing fairing dispensers have been recorded using onboard high speed cameras, whereas the rest of the tests have been tracked by external means.

The chase aircraft used for the test was A400M-0006 and the flares were ejected from A400M-0002.

\subsection{Chase cameras configuration}

The chase aircraft A400M-0006 has recorded the flares flying in parallel to A400M-0002.

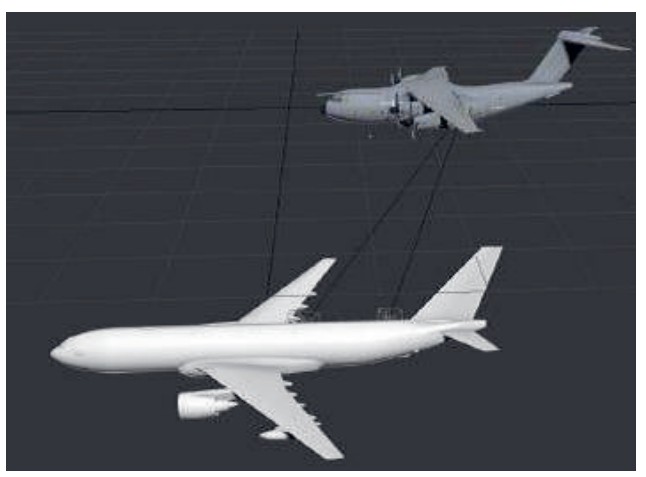

Figure 15.Chase cameras configuration

The two cameras installed are named Front Camera and Rear camera. Figures 16 and 17 show the images captured from these two cameras.

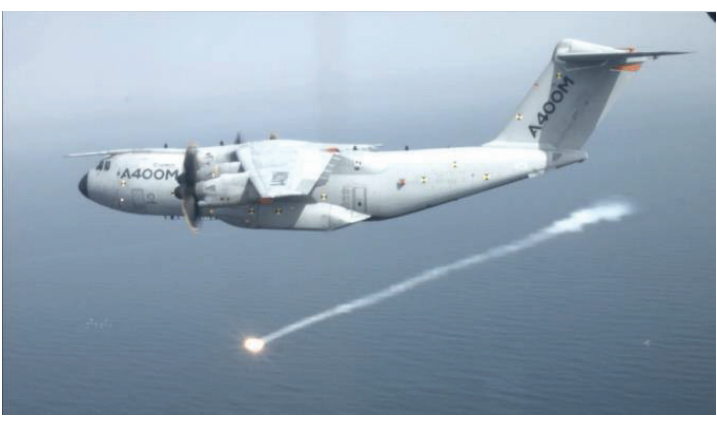

Figure 16. Front Camera

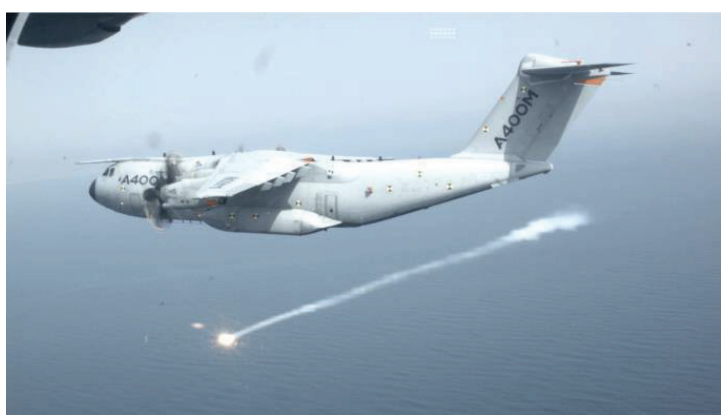

Figure 17. Rear camera

The cameras used in the test were two synchronized Blackmagic Micro Cinema Camera, fullHD (1920X1080 resolution), up to $60 \mathrm{fps}$.
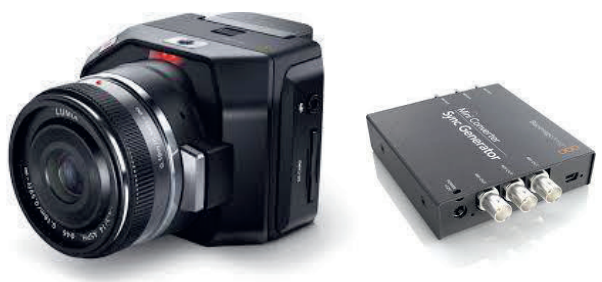

Figure 18. Blackmagic Micro Cinema Camera

\subsection{Analysis Methodology}

The flare trajectory is calculated by analyzing the recorded images using FollowMe software.

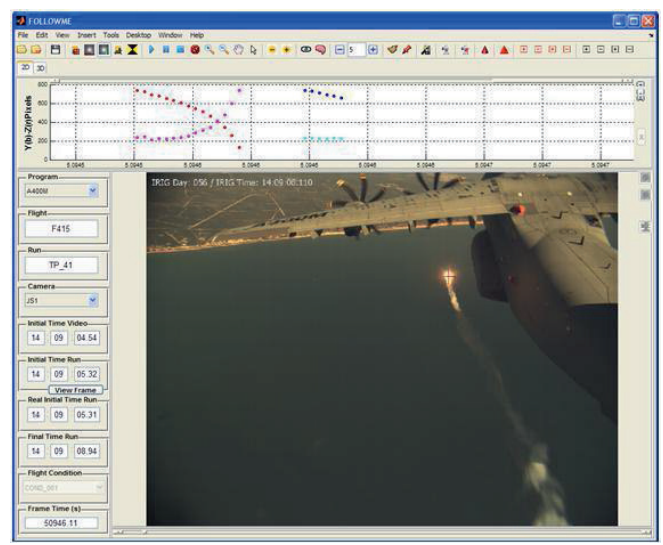

Figure 19.FollowMe interface 
The steps are the followings:

a) The cameras are positioned to respect A400M0006 Aircraft System Reference (chase AC).

b) The flares trajectories are calculated to respect A400M-0006 Aircraft System Reference (chase $\mathrm{AC})$.

c) The final trajectories are transformed from A400M-0002 to A400M-0006 Aircraft System Reference.

The first two steps are the same as the employed for onboard cameras configuration.

In the last step, the algorithm calculates the associated transformation matrix between both aircrafts for each frame.

To do so, the position of at least three points must be known in both axis systems for each instant time.

Several stickers are positioned with the aid of the tachymeter in the A400M-0002 so its coordinates are known in its axis reference system.

Following figure shows the stickers placed in the A400M-0002.

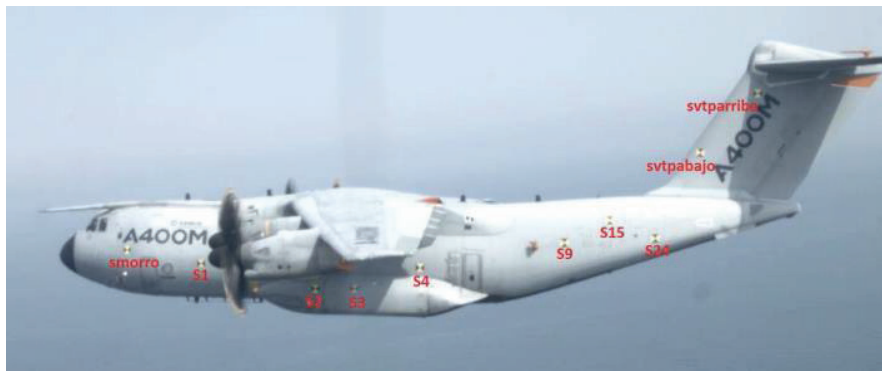

Figure 20. A400M-0002 stickers

With the knowledge of the position of the stickers in both systems, transformation matrix can be calculated and applied to the flares trajectories.

\subsection{Trajectory accuracy}

Sticker's positions do not change in A400M-0002 axis reference system. This information is used in the trajectory transformation process to obtain the accuracy of this transformation as well as of the photogrammetry tracking.
Next figure shows the stickers position deviation obtained during one flare trajectory.

For each sticker, the difference between the calculated position $(\mathrm{X}, \mathrm{Y}, \mathrm{Z})$ and the measured with tachymeter (in meters $)$ is represented $(<0.15 \mathrm{~m})$.

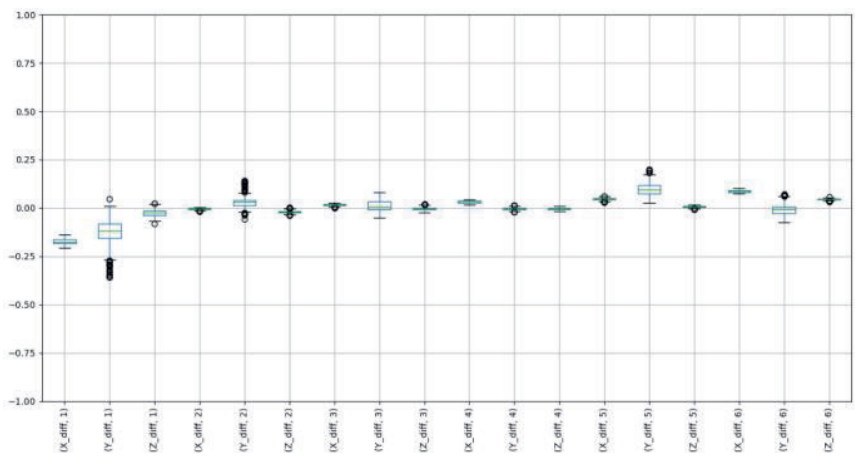

Figure 21. F0988-R025 stickers position deviation in meters

\section{Conclusions}

A new methodology has been implemented for safe separation analysis using external cameras installed in a chase aircraft.

The used of this technique is necessary when it is not possible to install onboard cameras to cover the entire trajectory.

This methodology has been used during the DASS system certification Flight Test campaign scheduled in May 2017.

\section{References}

[1] Coll Francisca , "A400M DASS. SYSTEM TEST", SFTE European Chapter Symposium 2015

[2] GML Lasermesstechnik. 3D Observer Operating Manual.Version 1.1.2005

[3] Camera calibration toolbox . http://www.vision.caltech.edu/bouguetj/calib_doc

\section{Acronyms}

DASS Defensive Aids Sub-System

HSV High Speed Video cameras 
HTP Horizontal Tail Plane

HS3 Camera installed on the balcony

J1S Camera installed under the left HTP

HS4 Camera installed on the left wing centre fuselage

WR Wing firing

SP Sponsors

AC Aircraft

FullHD High Definition (1920X1080) pixels

COT Camera of things 